\title{
Glycated Hemoglobin, Diabetes, and Cardiovascular Risk in Nondiabetic Adults
}

\author{
Elizabeth Selvin, Ph.D., M.P.H., Michael W. Steffes, M.D., Ph.D., Hong Zhu, B.S., \\ Kunihiro Matsushita, M.D., Ph.D., Lynne Wagenknecht, Dr.P.H., \\ James Pankow, Ph.D., M.P.H., Josef Coresh, M.D., Ph.D., \\ and Frederick L. Brancati, M.D., M.H.S.
}

From the Department of Epidemiology and the Welch Center for Prevention, Epidemiology, and Clinical Research (E.S., K.M., J.C., F.L.B.), and the Department of Biostatistics (H.Z., J.C.), Johns Hopkins Bloomberg School of Public Health; and the Division of General Internal Medicine, Department of Medicine, Johns Hopkins University (E.S., J.C., F.L.B.) - all in Baltimore; the Department of Laboratory Medicine and Pathology, Medical School (M.W.S.), and the Division of Epidemiology and Community Health (J.P.), University of Minnesota, Minneapolis; and the Division of Public Health Sciences, Wake Forest University School of Medicine, Winston-Salem, NC (L.W.). Address reprint requests to Dr. Selvin at Johns Hopkins Bloomberg School of Public Health, 2024 E. Monument St., Suite 2-600, Baltimore, MD 21287, or at Iselvin@ jhsph.edu.

N Engl J Med 2010;362:800-11.

Copyright (๑) 2010 Massachusetts Medical Society.

\section{A B STRACT}

\section{BACKGROUND}

Fasting glucose is the standard measure used to diagnose diabetes in the United States. Recently, glycated hemoglobin was also recommended for this purpose.

\section{METHODS}

We compared the prognostic value of glycated hemoglobin and fasting glucose for identifying adults at risk for diabetes or cardiovascular disease. We measured glycated hemoglobin in whole-blood samples from 11,092 black or white adults who did not have a history of diabetes or cardiovascular disease and who attended the second visit (occurring in the 1990-1992 period) of the Atherosclerosis Risk in Communities (ARIC) study.

\section{RESULTS}

The glycated hemoglobin value at baseline was associated with newly diagnosed diabetes and cardiovascular outcomes. For glycated hemoglobin values of less than $5.0 \%, 5.0$ to less than $5.5 \%, 5.5$ to less than $6.0 \%, 6.0$ to less than $6.5 \%$, and $6.5 \%$ or greater, the multivariable-adjusted hazard ratios (with $95 \%$ confidence intervals) for diagnosed diabetes were 0.52 (0.40 to 0.69 ), 1.00 (reference), 1.86 (1.67 to 2.08), 4.48 (3.92 to 5.13), and 16.47 (14.22 to 19.08), respectively. For coronary heart disease, the hazard ratios were 0.96 (0.74 to 1.24$), 1.00$ (reference), 1.23 (1.07 to 1.41), 1.78 (1.48 to 2.15), and 1.95 (1.53 to 2.48), respectively. The hazard ratios for stroke were similar. In contrast, glycated hemoglobin and death from any cause were found to have a J-shaped association curve. All these associations remained significant after adjustment for the baseline fasting glucose level. The association between the fasting glucose levels and the risk of cardiovascular disease or death from any cause was not significant in models with adjustment for all covariates as well as glycated hemoglobin. For coronary heart disease, measures of risk discrimination showed significant improvement when glycated hemoglobin was added to models including fasting glucose.

\section{CONCLUSIONS}

In this community-based population of nondiabetic adults, glycated hemoglobin was similarly associated with a risk of diabetes and more strongly associated with risks of cardiovascular disease and death from any cause as compared with fasting glucose. These data add to the evidence supporting the use of glycated hemoglobin as a diagnostic test for diabetes. 
F ASTING GLUCOSE IS THE STANDARD MEAsure used for the diagnosis of diabetes in the United States. ${ }^{1,2}$ Historically, glycated hemoglobin has been recommended only for the determination of glucose control among persons who have already received the diagnosis of diabetes. New clinical practice recommendations from the American Diabetes Association advocate the use of glycated hemoglobin in the diagnosis of diabetes, largely on the basis of the established association between glycated hemoglobin and microvascular disease. ${ }^{3}$ Compared with fasting glucose, glycated hemoglobin has several advantages as a diagnostic test: it has higher repeatability, ${ }^{4-6}$ can be assessed in the nonfasting state, and is the preferred test for monitoring glucose control. ${ }^{1}$ Longterm prognostic data are also useful for informing diagnostic cutoff points for asymptomatic conditions, and there is evidence that elevated glycated hemoglobin values may be a risk factor for macrovascular disease.

This study was designed to characterize and compare the relationships between values of glycated hemoglobin and fasting glucose and the risk of diabetes, coronary heart disease, ischemic stroke, and death from any cause in a large community-based cohort of middle-aged adults who did not have a history of diabetes. We also investigated whether the association of glycated hemoglobin with newly diagnosed cardiovascular disease could be explained by the intervening development of diabetes. We hypothesized that glycated hemoglobin would be superior to fasting glucose as an indicator of risk for the development of diabetes and cardiovascular disease and for death, with possible differences on the basis of race or ethnic group. Blacks who have diabetes are well known to have higher glycated hemoglobin values than their white counterparts; the same disparity holds among nondiabetic adults. ${ }^{7-10}$ However, the clinical implications of these disparities are unknown, and few data exist on glycated hemoglobin and outcomes among blacks.

\section{METHODS}

\section{STUDY POPULATION}

The Atherosclerosis Risk in Communities (ARIC) study is a community-based prospective cohort study of 15,792 middle-aged adults from four U.S. communities. The first examination of participants (visit 1) took place during the 1987-1989 period, with three follow-up visits taking place, each approximately every 3 years. ${ }^{11,12}$ Visit 2 (during 1990-1992), attended by 14,348 participants, was the only visit for which stored whole-blood samples were available for measurement of glycated hemoglobin; this was the baseline visit in the present study. We excluded participants who identified themselves as other than white or black, as well as those who had self-reported diabetes or use of diabetes medication (as recorded during visit 1 or visit 2), or a history of cardiovascular disease (as recorded during visit 1 or visit 2) or a validated cardiovascular event between visit 1 and visit 2 or who were in a nonfasting state or had missing data. Our final sample size was 11,092 persons. Institutional review boards at each clinical site approved the study protocol, and written informed consent was obtained from all participants.

\section{MEASUREMENT OF GLYCATED HEMOGLOBIN}

We thawed and assayed frozen whole-blood samples collected at ARIC visit 2 for the measurement of glycated hemoglobin using high-performance liquid chromatography (with the use of the Tosoh A1c 2.2 Plus Glycohemoglobin Analyzer method in 2003-2004 and the Tosoh G7 method in 20072008, Tosoh Corp). (Both instruments were standardized to the Diabetes Control and Complications Trial assay.)

\section{ASSESSMENT OF DIABETES}

The serum glucose level was measured by means of the hexokinase method. We used two definitions of newly identified diabetes: a visit-based definition and an interview-based definition. Visitbased diabetes was defined according to a standard time-to-diabetes definition based on glucose measurements, a self-reported diagnosis of diabetes, or medication use for a maximum of 6 years of follow-up. ${ }^{13}$ Interview-based diabetes was defined on the basis of a self-reported diabetes diagnosis or diabetes medication use during the ARIC visits and subsequent annual telephone calls for a maximum of 15 years of follow-up.

\section{OTHER VARIABLES OF INTEREST}

Plasma lipid level, ${ }^{14-17}$ body-mass index (BMI), waist-to-hip ratio, ${ }^{18}$ and blood pressure ${ }^{19}$ were measured according to the published methods. Hypertension was defined as the average of two bloodpressure readings at the visit (with systolic blood pressure having a cutoff point of $140 \mathrm{~mm} \mathrm{Hg}$ or 
higher and diastolic blood pressure having a cutoff point of $90 \mathrm{~mm} \mathrm{Hg}$ or higher) or the use of hypertension medication. Participants reported their education level, alcohol use, and smoking status. The level of physical activity was assessed with the use of Baecke's questionnaire at ARIC visit $1 .^{20}$

SURVEILLANCE FOR NEWLY DIAGNOSED CORONARY HEART DISEASE, STROKE, AND DEATH FROM ANY CAUSE

The ascertainment of deaths and classification of cardiovascular events are detailed elsewhere. ${ }^{21,22}$ Briefly, potential cardiovascular hospitalizations were reported annually by participants and also identified through community-wide hospital surveillance. Trained personnel abstracted hospital records related to possible cardiovascular events. ${ }^{22}$ Silent myocardial infarctions, as detected by means of electrocardiography during the visits, were identified and recorded. We defined newly diagnosed coronary heart disease as a definite or probable myocardial infarction, a death from coronary heart disease, a cardiac procedure, or electrocardiographic evidence of a silent myocardial infarction. We also examined definite or probable ischemic stroke. Adjudicated follow-up data for cardiovascular events were available up to January 1, 2006.

\section{STATISTICAL ANALYSIS}

Baseline characteristics of the study population (from ARIC visit 2) were calculated both overall and according to categories of glycated hemoglobin values $(<5.0 \%, 5.0$ to $<5.5 \%, 5.5$ to $<6.0 \%, 6.0$ to $<6.5 \%$, and $\geq 6.5 \%$ ). Adjusted hazard ratios and corresponding $95 \%$ confidence intervals were estimated with the use of Cox proportional-hazards models. The three core models were as follows: Model 1 was adjusted for age, sex, and race. Model 2 was adjusted for age, sex, race, low-density and high-density cholesterol levels, triglyceride level, BMI, waist-to-hip ratio, hypertension, family history of diabetes, education level, alcohol use, physical activity, and smoking status. We evaluated models 1 and 2 with regard to either the glycated hemoglobin categories (called models 1a and 2a) or standard fasting glucose categories $(<100,100$ to $<126$, and $\geq 126 \mathrm{mg}$ per deciliter [5.6, 5.6 to $<7.0$, and $\geq 7.0 \mathrm{mmol}$ per liter, respectively]) (called models $1 \mathrm{~b}$ and $2 \mathrm{~b}$ ). Finally, model 3 was adjusted for all the variables in model 2 plus either the baseline fasting glucose level (model 3a) or the baseline glycated hemoglobin value (model 3b).

The glycated hemoglobin category of 5.0 to less than $5.5 \%$ had the largest number of participants
(4950) and was used as the reference category. To assess the continuous associations between the glycated hemoglobin value and clinical outcomes in the models, we generated piecewise linear splines with knots corresponding to the glycated hemoglobin cutoff points used in this study; we also implemented restricted cubic splines to obtain a smoother fit to the data. Model discrimination was assessed with the use of Harrell's C statistic. ${ }^{23}$

We tested for interactions with race and sex. To investigate whether a diagnosis of diabetes made before a cardiovascular event or death could explain potential associations with glycated hemoglobin, we conducted analyses in which cases of diagnosed diabetes occurring before the event of interest were censored. We also conducted analyses examining the association of each outcome with the baseline glycated hemoglobin value after excluding persons with a fasting glucose level of $126 \mathrm{mg}$ per deciliter or higher at either ARIC visit 1 or 2. To assess whether glycated hemoglobin was associated with a risk of the outcomes across categories of fasting glucose at baseline, we conducted analyses of combined categories of glycated hemoglobin and fasting glucose. To evaluate the overall improvement in risk classification for the addition of glycated hemoglobin to fully adjusted models including fasting glucose, we calculated the net-reclassification-improvement statistic and the integrated-discrimination-improvement statistic. $^{24}$

All reported P values are two-sided. They are not adjusted for multiple comparisons.

\section{RESULTS}

Baseline characteristics of the study population, both overall and according to the glycated hemoglobin category, are shown in Table 1. Glycated hemoglobin and fasting glucose levels at baseline were highly correlated $(r=0.73)$. Participants with elevated glycated hemoglobin levels were more likely to be black than white, and to have fewer years of education, and to have an adverse lipid profile and a higher BMI and were less likely to be current drinkers than to have formerly or never used alcohol. As documented in previous studies, in our nondiabetic population, blacks had significantly higher mean glycated hemoglobin values $(5.8 \%)$ than whites $(5.4 \%)(\mathrm{P}<0.001)$.

The median follow-up time was approximately 14 years. More than $80 \%$ of the participants at- 


\begin{tabular}{|c|c|c|c|c|c|c|}
\hline \multirow[t]{2}{*}{ Value } & \multicolumn{6}{|c|}{ Glycated Hemoglobin Category } \\
\hline & $\begin{array}{c}\text { Any } \\
(\mathrm{N}=11,092)\end{array}$ & $\begin{array}{c}<5.0 \% \\
(N=949)\end{array}$ & $\begin{array}{c}5.0 \text { to }<5.5 \% \\
(\mathrm{~N}=4950)\end{array}$ & $\begin{array}{c}5.5 \text { to }<6.0 \% \\
(\mathrm{~N}=3683)\end{array}$ & $\begin{array}{c}6.0 \text { to }<6.5 \% \\
(N=1031)\end{array}$ & $\begin{array}{c}\geq 6.5 \% \\
(\mathrm{~N}=479)\end{array}$ \\
\hline Glycated hemoglobin (\%) & $5.5 \pm 0.6$ & $4.8 \pm 0.2$ & $5.2 \pm 0.1$ & $5.7 \pm 0.1$ & $6.1 \pm 0.1$ & $7.4 \pm 1.4$ \\
\hline Fasting glucose (mg/dl) & $104.7 \pm 18.6$ & $98.0 \pm 8.8$ & $99.7 \pm 9.4$ & $104.5 \pm 10.6$ & $113.4 \pm 15.5$ & $153.1 \pm 51.7$ \\
\hline \multicolumn{7}{|l|}{ Fasting glucose category (\%) } \\
\hline$<100 \mathrm{mg} / \mathrm{dl}$ & 41.3 & 60.5 & 53.2 & 32.8 & 14.9 & 1.7 \\
\hline 100 to $<126 \mathrm{mg} / \mathrm{dl}$ & 52.4 & 38.7 & 45.7 & 64.2 & 67.2 & 27.8 \\
\hline$\geq 126 \mathrm{mg} / \mathrm{dl} \uparrow$ & 6.3 & 0.8 & 1.1 & 3.0 & 17.9 & 70.6 \\
\hline Age (yr) & $56.7 \pm 5.7$ & $55.3 \pm 5.5$ & $56.1 \pm 5.6$ & $57.3 \pm 5.7$ & $58.0 \pm 5.7$ & $57.6 \pm 5.7$ \\
\hline \multicolumn{7}{|l|}{$\operatorname{Sex}(\%)$} \\
\hline Female & 57.7 & 55.2 & 58.8 & 56.8 & 55.8 & 61.8 \\
\hline Male & 42.3 & 44.8 & 42.2 & 43.2 & 44.2 & 38.2 \\
\hline \multicolumn{7}{|l|}{ Race $(\%) \ddagger$} \\
\hline Black & 22.4 & 15.5 & 11.9 & 27.0 & 49.1 & 52.2 \\
\hline White & 77.6 & 84.5 & 88.1 & 73.0 & 50.9 & 47.8 \\
\hline \multicolumn{7}{|l|}{ Fasting cholesterol (mg/dl) } \\
\hline LDL & $133.0 \pm 36.4$ & $122.8 \pm 34.7$ & $130.0 \pm 34.9$ & $136.6 \pm 37.0$ & $138.6 \pm 37.5$ & $143.6 \pm 39.0$ \\
\hline HDL & $50.9 \pm 16.7$ & $53.2 \pm 18.5$ & $52.5 \pm 17.0$ & $50.1 \pm 16.2$ & $47.0 \pm 14.7$ & $43.9 \pm 13.6$ \\
\hline \multicolumn{7}{|l|}{ Fasting triglycerides $(\mathrm{mg} / \mathrm{dl})$} \\
\hline Median & 110 & 101 & 105 & 111 & 121 & 139 \\
\hline Interquartile range & $80-154$ & $73-136$ & $78-150$ & $81-155$ & $88-164$ & 99-190 \\
\hline Body-mass index $\mathbb{}$ & $27.7 \pm 5.3$ & $26.5 \pm 4.7$ & $26.7 \pm 4.6$ & $28.0 \pm 5.3$ & $30.0 \pm 6.0$ & $32.5 \pm 6.3$ \\
\hline Waist-to-hip ratio & $0.9 \pm 0.1$ & $0.9 \pm 0.1$ & $0.9 \pm 0.1$ & $0.9 \pm 0.1$ & $0.9 \pm 0.1$ & $1.0 \pm 0.1$ \\
\hline Hypertension (\%) & 32.0 & 26.9 & 26.7 & 33.8 & 49.4 & 56.8 \\
\hline Family history of diabetes (\%) & 22.7 & 19.5 & 20.4 & 23.9 & 27.1 & 33.8 \\
\hline \multicolumn{7}{|l|}{ Education (\%) } \\
\hline Less than high school & 19.2 & 13.0 & 14.0 & 22.6 & 31.7 & 33.2 \\
\hline High school or equivalent & 42.0 & 40.6 & 44.5 & 41.1 & 37.3 & 36.1 \\
\hline College or above & 38.8 & 46.4 & 41.5 & 36.3 & 31.0 & 30.7 \\
\hline \multicolumn{7}{|l|}{ Alcohol use (\%) } \\
\hline Currently & 59.3 & 64.3 & 64.9 & 56.4 & 47.3 & 40.3 \\
\hline Formerly & 18.6 & 16.7 & 15.1 & 20.2 & 26.6 & 27.8 \\
\hline Never & 22.1 & 19.1 & 20.0 & 23.4 & 26.1 & 31.9 \\
\hline Baecke's physical-activity index score & $2.5 \pm 0.8$ & $2.5 \pm 0.8$ & $2.5 \pm 0.8$ & $2.4 \pm 0.8$ & $2.3 \pm 0.7$ & $2.3 \pm 0.7$ \\
\hline \multicolumn{7}{|l|}{ Smoking status (\%) } \\
\hline Current smoker & 21.9 & 13.0 & 18.0 & 27.5 & 29.0 & 22.3 \\
\hline Former smoker & 37.3 & 42.6 & 39.0 & 34.5 & 34.0 & 37.0 \\
\hline Never smoked & 40.8 & 44.4 & 43.0 & 38.0 & 37.0 & 40.7 \\
\hline
\end{tabular}

* Plus-minus values are means \pm SD. To convert the values for fasting glucose to millimoles per liter, multiply by 0.05551 . To convert the values for cholesterol to millimoles per liter, multiply by 0.02586 . To convert the values for triglycerides to millimoles per liter, multiply by 0.01129 . HDL denotes high-density lipoprotein, and LDL low-density lipoprotein.

$\dagger$ The 699 persons $(6.3 \%)$ with a fasting glucose level of $126 \mathrm{mg}$ per deciliter $(7 \mathrm{mmol}$ per liter) or higher at baseline $(8,56,112,185$, and 338 persons with a glycated hemoglobin category of $<5.0 \%, 5.0$ to $<5.5 \%, 5.5$ to $<6.0 \%, 6.0$ to $<6.5 \%$, and $\geq 6.5 \%$, respectively) were excluded from the visit-based analysis examining the risk of diabetes with the use of fasting glucose levels during the first 6 years of follow-up but were included in the interview-based analysis of diagnosed diabetes during the 15 years of follow-up.

$t$ Race was self-reported.

$\int$ The body-mass index is the weight in kilograms divided by the square of the height in meters.

I Baecke's physical-activity index is measured with the use of a questionnaire about leisure-time sports activities developed by Baecke et al. ${ }^{20}$ The scale ranges from 1 to 4 , with a score of 4 indicating the greatest activity. 
tended each clinic visit, and retention of ARIC participants during the follow-up period was high $(>90 \%)$. During the first 6 years of follow-up, 620 participants were identified as having diabetes, according to the visit-based diabetes definition. During the full 15 years of follow-up, 2251 participants reported a diagnosis of diabetes or use of diabetes medication (the interview-based definition of diabetes), there were 1198 validated cases of coronary heart disease and 358 validated cases of ischemic stroke, and 1447 participants died.

The crude cumulative 15-year incidence (calculated as the incidence proportion) of self-reported diabetes was $20 \%$. (See the Supplementary Appendix, available with the full text of this article at NEJM.org, for a discussion of self-reported diabetes vs. verified diabetes.) The cumulative incidence of diagnosed diabetes was $6 \%, 12 \%, 21 \%, 44 \%$, and $79 \%$ among participants with a glycated hemoglobin value of less than $5.0 \%, 5.0$ to less than $5.5 \%, 5.5$ to less than $6.0 \%, 6.0$ to less than $6.5 \%$, and $6.5 \%$ or higher, respectively. The incidence rates (per 1000 person-years) are shown according to the category of glycated hemoglobin in Figure 1. The adjusted hazard ratios (and 95\% confidence intervals) of the 6-year risk of visit-based diabetes and 15-year risk of the other outcomes are shown in Table 2 according to the category of glycated hemoglobin. A baseline glycated hemoglobin value of less than $5.0 \%$, as compared with a value of 5.0 to less than $5.5 \%$, was associated with approximately half the risk of visit-based or diagnosed diabetes (hazard ratios for each outcome in models 1 and 2, approximately 0.50). Additional adjustment for the fasting glucose level (in model 3a) attenuated this association, but the glycated hemoglobin category remained strongly associated with diagnosed diabetes. We also observed significant trends of an increasing risk of coronary heart disease, ischemic stroke, and death from any cause with higher levels of baseline glycated hemoglobin. These associations persisted even after adjustment for fasting glucose (model $3 a$ ).

Figure 2 depicts the adjusted hazard ratios for diagnosed diabetes, coronary heart disease, stroke, and death from any cause and the baseline glycated hemoglobin category - per each absolute increase of 1 percentage point in the glycated hemoglobin value. There was no evidence of a threshold value of glycated hemoglobin for diagnosed diabetes, but there was evidence for a possible threshold for the risk of coronary heart disease. There was no significant association between the glycated hemoglobin value and the risk of coronary heart disease at glycated hemoglobin values of less than $5.0 \%$, but as compared with that category, glycated hemoglobin values of $5.5 \%$ or higher were associated with a hazard ratio for coronary heart disease of 1.38 (95\% confidence interval [CI], 1.22 to 1.56). For death from any cause, we observed a J-shaped association. Participants with glycated hemoglobin values in the lowest category $(<5.0 \%)$ had a significantly higher risk of death from any cause as compared with those with glycated hemoglobin levels of 5.0 to less than 5.5\% (Table 2 and Fig. 2). In post hoc analyses, the J-shaped association persisted after the exclusion of participants with anemia, adjustment for hematocrit level and mean corpuscular volume, and the exclusion of deaths that occurred during the first 3 years of follow-up (data not shown). In analyses censoring data for persons who received a diagnosis of diabetes during the follow-up period, the associations remained significant between glycated hemoglobin and coronary heart disease, stroke, and death from any cause.

The fasting glucose categories were associated with the risks of outcomes in the minimally adjusted models, but these associations were attenuated after adjustment for other risk factors (Table 3). As compared with a baseline fasting glucose level of less than $100 \mathrm{mg}$ per deciliter, a level of 100 to less than 126 mg per deciliter was associated with diagnosed diabetes (hazard ratio, 2.31; 95\% CI, 2.06 to 2.59) but not with coronary heart disease (hazard ratio, 1.03; 95\% CI, 0.91 to 1.18), ischemic stroke (hazard ratio, $0.97 ; 95 \% \mathrm{CI}$, 0.76 to 1.23 ), and death from any cause (hazard ratio, 1.07; 95\% CI, 0.96 to 1.21) after adjustment for covariates (model 2b) (Table 3), whereas undiagnosed diabetes (defined as a fasting glucose level of $126 \mathrm{mg}$ per deciliter or higher at baseline) was significantly, independently associated with the development of coronary heart disease (hazard ratio, 1.29; 95\% CI, 1.04 to 1.61), ischemic stroke (hazard ratio, 1.89; 95\% CI, 1.33 to 2.69 ), and death from any cause (hazard ratio, 1.31; $95 \%$ CI, 1.07 to 1.61). After additional adjustment for glycated hemoglobin in model $3 \mathrm{~b}$, there was no significant association between fasting glucose category and the risk of coronary heart disease, ischemic stroke, or death from any cause. Among 
the 10,069 participants with a fasting glucose level of less than $126 \mathrm{mg}$ per deciliter at visit 1 and visit 2, the glycated hemoglobin category was similarly associated, in model $3 \mathrm{~b}$ as compared with models $1 \mathrm{~b}$ and $2 \mathrm{~b}$, with diagnosed diabetes, coronary heart disease, stroke, and death from any cause (Table 2 in the Supplementary Appendix).

We also assessed the associations of three categories of glycated hemoglobin $(<6.0 \%, 6.0$ to $<6.5 \%$, and $\geq 6.5 \%$ ) with the risks of outcomes among participants stratified according to the fasting glucose category $(<100,100$ to $<126$, and $\geq 126$ mg per deciliter) (Table 3 in the Supplementary Appendix). The glycated hemoglobin categories of 6.0 to less than $6.5 \%$ and $6.5 \%$ or higher were significantly associated with all outcomes within each fasting glucose category, with the association increasing with higher glycated hemoglobin categories. In contrast, if the glycated hemoglobin value was less than $6.0 \%$, fasting glucose was not significantly associated with coronary heart disease, ischemic stroke, or death from any cause.

There was no significant interaction between sex and glycated hemoglobin category for any of the clinical outcomes ( $P>0.20$ for all interactions). There was also no significant interaction between race and glycated hemoglobin value regarding the risk of coronary heart disease, ischemic stroke, or death from any cause ( $\mathrm{P}>0.80$ for all interactions). However, race did appear to modify the association between the glycated hemoglobin value and the risk of diagnosed diabetes during 15 years of follow-up ( $\mathrm{P}=0.007$ for interaction), but there was no interaction between race and the association between glycated hemoglobin value and the 6-year risk of visit-based diabetes $(\mathrm{P}=0.81$ for interaction). As compared with whites, blacks had lower adjusted hazard ratios for reporting a diagnosis of diabetes during the 15 years of follow-up, within each category of glycated hemoglobin. A similar interaction was also observed between race and fasting glucose level regarding the risk of self-reported diabetes $(\mathrm{P}=0.01$ for interaction).

The net-reclassification-improvement and integrated-discrimination-improvement statistics for diagnosed diabetes and coronary heart disease were significantly improved with the addition of glycated hemoglobin (modeled continuously) to the model of fasting glucose (as a continuous variable) and other covariates (see the Supplementary Appendix).

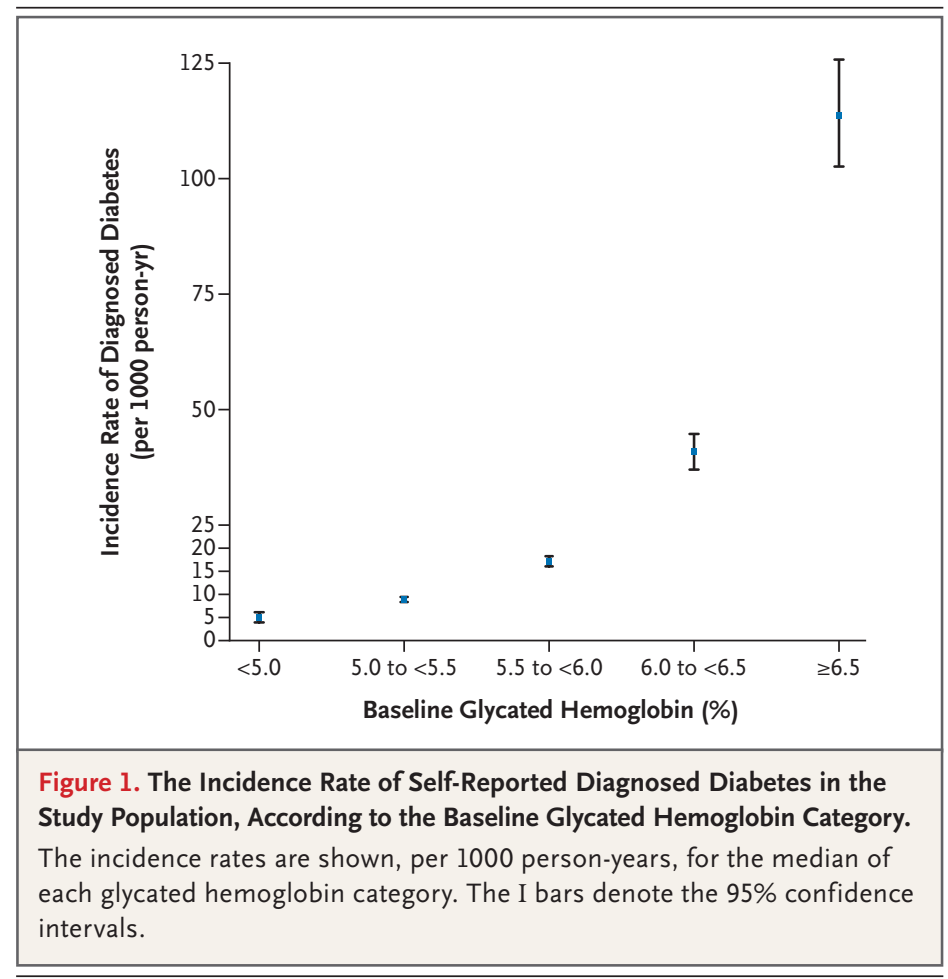

\section{DISCUSSION}

Among the people in the United States who do not have a diagnosis of diabetes, over 2.4 million have a glycated hemoglobin value higher than $6.5 \%$ and 7 million have a value higher than $6.0 \% .^{7}$ Our findings show that people with a glycated hemoglobin value of $6.0 \%$ or higher are at high risk for the development of diabetes, even after adjustment for other risk factors and independently of baseline fasting glucose levels. We also observed that glycated hemoglobin is a marker of cardiovascular risk. In this nondiabetic population, glycated hemoglobin remained associated with cardiovascular disease and death even after we accounted for baseline fasting glucose levels; in contrast, fasting glucose was not significantly associated after adjustment for the glycated hemoglobin value. We also demonstrated improved risk reclassification for coronary heart disease with the inclusion of glycated hemoglobin in fully adjusted models, suggesting that glycated hemoglobin may be superior to fasting glucose for characterizing longterm risk.

Glycated hemoglobin values reflect the 2-to-3month average endogenous exposure to glucose, including postprandial spikes in the blood glucose level, and have low intraindividual variability, par- 
Table 2. Adjusted Hazard Ratios for Selected Clinical Outcomes in the Study Population during the 15-Year Study Period, According to the Glycated Hemoglobin Category at Baseline and the Model.*

\section{Outcome}

Model la

Model 2a

Model 3a

\section{Visit-based diabetes $\hat{\dagger}$}

Glycated hemoglobin category — hazard ratio $(95 \% \mathrm{Cl})$

$$
\begin{aligned}
& <5.0 \% \\
& 5.0 \text { to }<5.5 \% \text { (reference) } \\
& 5.5 \text { to }<6.0 \% \\
& 6.0 \text { to }<6.5 \% \\
& \geq 6.5 \%
\end{aligned}
$$

$P$ value for trend

Glycated hemoglobin value - hazard ratio $(95 \% \mathrm{Cl})$

C statistic

\section{Diagnosed diabetes:}

Glycated hemoglobin category — hazard ratio $(95 \% \mathrm{Cl})$

$$
\begin{aligned}
& <5.0 \% \\
& 5.0 \text { to }<5.5 \% \text { (reference) } \\
& 5.5 \text { to }<6.0 \% \\
& 6.0 \text { to }<6.5 \% \\
& \geq 6.5 \%
\end{aligned}
$$

$P$ value for trend

Glycated hemoglobin value — hazard ratio $(95 \% \mathrm{Cl})$

C statistic

\section{Coronary heart disease}

Glycated hemoglobin category — hazard ratio $(95 \% \mathrm{Cl})$

$$
\begin{aligned}
& <5.0 \% \\
& 5.0 \text { to }<5.5 \% \text { (reference) } \\
& 5.5 \text { to }<6.0 \% \\
& 6.0 \text { to }<6.5 \% \\
& \geq 6.5 \%
\end{aligned}
$$

$P$ value for trend

Glycated hemoglobin value — hazard ratio $(95 \% \mathrm{Cl})$

C statistic

\section{Ischemic stroke}

Glycated hemoglobin category — hazard ratio $(95 \% \mathrm{Cl})$

$$
\begin{aligned}
& <5.0 \% \\
& 5.0 \text { to }<5.5 \% \text { (reference) } \\
& 5.5 \text { to }<6.0 \% \\
& 6.0 \text { to }<6.5 \% \\
& \geq 6.5 \%
\end{aligned}
$$

$P$ value for trend

Glycated hemoglobin value - hazard ratio $(95 \% \mathrm{Cl})$

C statistic

$\begin{array}{cc}0.49(0.27-0.89) & 0.50(0.28-0.90) \\ 1.00 & 1.00 \\ 2.91(2.33-3.63) & 2.44(1.95-3.05) \\ 13.38(10.51-17.03) & 9.20(7.18-11.78) \\ 50.73(37.44-68.74) & 32.77(23.96-44.82) \\ <0.001 & <0.001 \\ 2.73(2.56-2.91) & 2.75(2.55-2.96) \\ 0.7771 & 0.8258\end{array}$

$0.57(0.31-1.03)$

1.00

$1.77(1.41-2.22)$

$5.08(3.93-6.56)$

$14.53(10.53-20.04)$

$<0.001$

$2.57(2.35-2.81)$

0.8695

$\begin{array}{llc}0.51(0.39-0.67) & 0.52(0.40-0.69) & 0.53(0.40-0.69) \\ 1.00 & 1.00 & 1.00 \\ 2.12(1.90-2.37) & 1.86(1.67-2.08) & 1.80(1.61-2.01) \\ 6.29(5.52-7.17) & 4.48(3.92-5.13) & 4.03(3.52-4.61) \\ 27.19(23.61-31.31) & 16.47(14.22-19.08) & 10.40(8.80-12.28) \\ <0.001 & <0.001 & <0.001 \\ 1.97(1.92-2.03) & 1.80(1.75-1.86) & 1.44(1.35-1.55) \\ 0.7458 & 0.7766 & 0.7816\end{array}$

$\begin{array}{lll}0.89(0.69-1.15) & 0.96(0.74-1.24) & 0.95(0.73-1.22) \\ 1.00 & 1.00 & 1.00 \\ 1.45(1.27-1.66) & 1.23(1.07-1.41) & 1.25(1.09-1.44) \\ 2.37(1.98-2.84) & 1.78(1.48-2.15) & 1.88(1.55-2.28) \\ 2.91(2.31-3.67) & 1.95(1.53-2.48) & 2.46(1.84-3.28) \\ <0.001 & <0.001 & <0.001 \\ 1.34(1.27-1.42) & 1.19(1.11-1.27) & 1.50(1.33-1.68) \\ \quad 0.6888 & 0.7351 & 0.7383\end{array}$

$\begin{array}{llc}1.06(0.65-1.71) & 1.09(0.67-1.76) & 1.09(0.68-1.77) \\ 1.00 & 1.00 & 1.00 \\ 1.27(0.97-1.67) & 1.17(0.89-1.53) & 1.16(0.89-1.53) \\ 2.63(1.92-3.61) & 2.22(1.60-3.08) & 2.19(1.58-3.05) \\ 3.68(2.56-5.30) & 3.16(2.15-4.64) & 2.96(1.87-4.67) \\ <0.001 & <0.001 & <0.001 \\ 1.41(1.30-1.54) & 1.34(1.22-1.48) & 1.55(1.28-1.88) \\ 0.7229 & 0.7581 & 0.7594\end{array}$




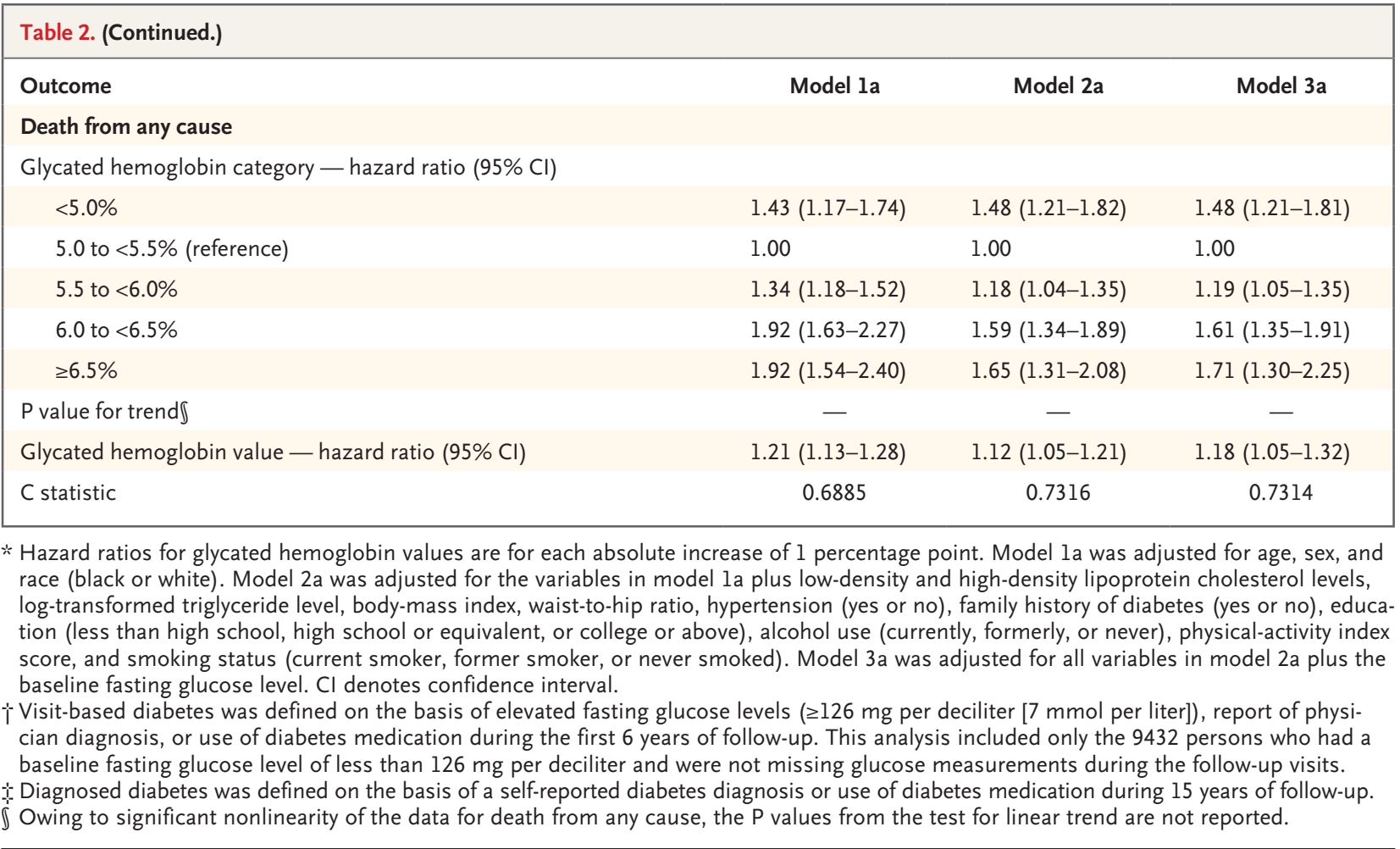

ticularly in persons without diabetes. ${ }^{4,25}$ These characteristics may contribute to the superiority of glycated hemoglobin over fasting glucose for long-term macrovascular risk stratification.

Recommendations for the diagnosis of diabetes are based on the relations of fasting glucose and glycated hemoglobin with microvascular disease, typically retinopathy. ${ }^{1,3}$ Nonetheless, cardiovascular disease is the leading cause of illness, death, and hospitalization in persons with diabetes. ${ }^{26,27}$ Our data suggest that glycated hemoglobin values in the normal range can identify persons at increased risk for coronary heart disease, stroke, and death before the diagnosis of diabetes, indicating that glycated hemoglobin is a useful marker of cardiovascular risk and death from any cause. The J-shaped relation between the glycated hemoglobin value and the risk of death from any cause suggests that further exploration of the health risks associated with the low-normal glycemic state and possible nonglycemic determinants of glycated hemoglobin is warranted.

As in the present study, the literature has documented an increase in cardiovascular risk with increases in glycated hemoglobin values within the nondiabetic range. ${ }^{28-36}$ We have previously shown (using a case-cohort design) associations of glycated hemoglobin with coronary heart disease and stroke in a subgroup of the ARIC population with low fasting glucose levels (at two time points) and low glycated hemoglobin values ${ }^{37}$ and, separately, among persons with diabetes. ${ }^{38}$ Nonetheless, recent clinical trials have shown little benefit, and possibly some harm, of lowering the glycated hemoglobin value in patients with diabetes to prevent cardiovascular outcomes. ${ }^{39-43}$ In contrast, the microvascular benefits of glucose control are well established. ${ }^{44,45}$ Although the causal role of glucose itself in the development of cardiovascular disease is unclear, our data demonstrate that glycated hemoglobin within the normal range can be a useful marker of cardiovascular risk. Therefore, glycated hemoglobin values exceeding $6.0 \%$ may be a clinically useful marker to identify persons at risk for the development of not only diabetes but also cardiovascular disease and death.

Despite significant differences between blacks and whites in glycated hemoglobin values at baseline, race did not modify the associations between glycated hemoglobin value and cardiovascular outcomes and death in this population. We did, how- 


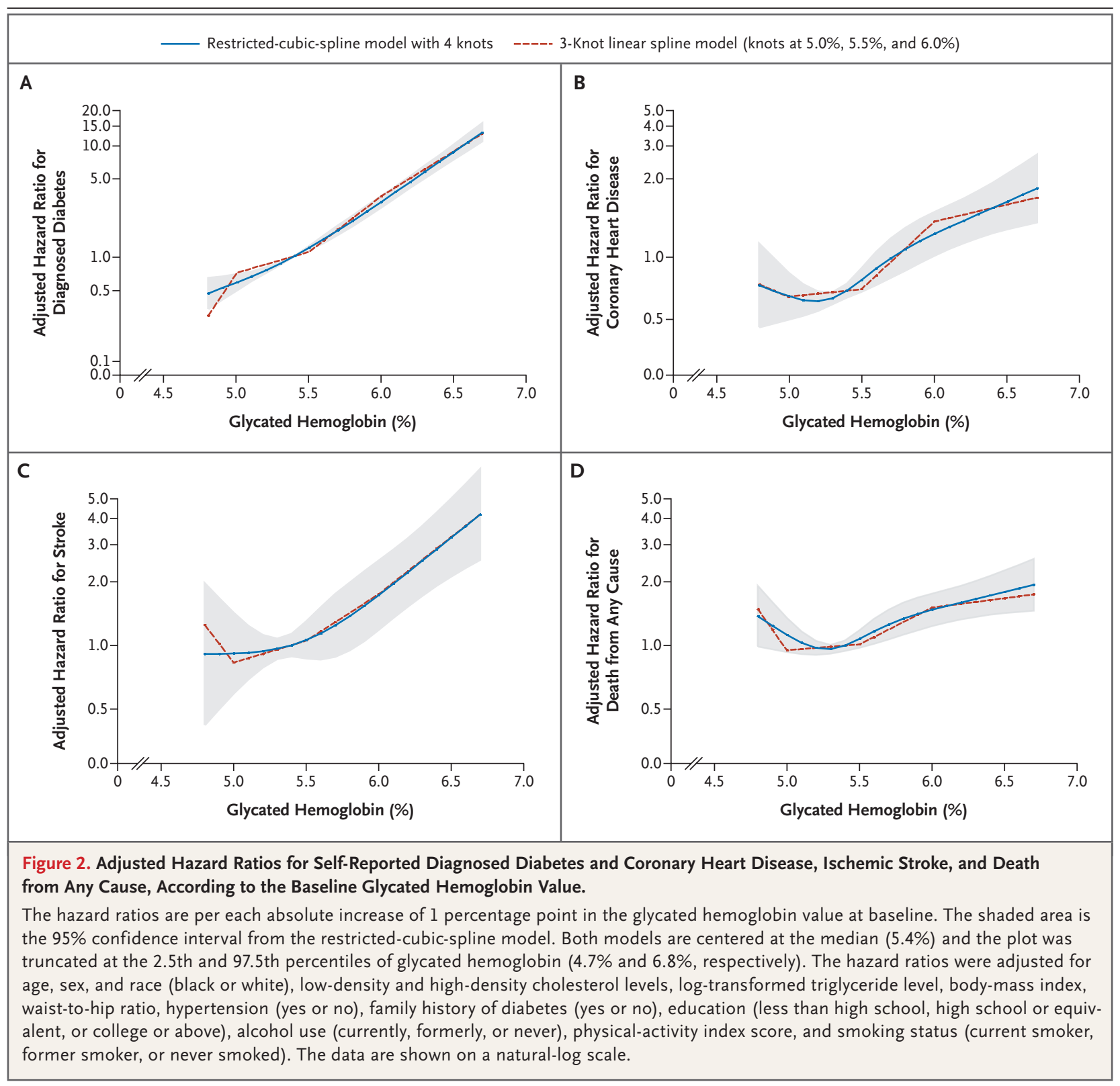

ever, observe a significant interaction between race and diagnosed diabetes during the 15 years of follow-up (but not for the 6-year visit-based definition), revealing that blacks were significantly less likely than whites to report a diabetes diagnosis, across all categories of glycated hemoglobin. We observed a similar race interaction for baseline fasting glucose level and subsequent diagnosis of diabetes. Thus, higher values of glycated hemoglobin in nondiabetic blacks as compared with nondiabetic whites might be partially explained by delays in diagnosis. Our findings do not support the use of race-specific glycated hemoglobin cutoff points for the identification of persons at risk for diabetes, cardiovascular disease, or death.

Important limitations of this study include the reliance on single glycated hemoglobin and glucose measurements at baseline, a limited number of fasting glucose measurements during the follow-up period, and the lack of validation of selfreported diabetes for the 15-year analyses. Nonetheless, among sensitivity analyses comparing diabetes definitions based on fasting glucose level, medication use, and self-reported information - 
Table 3. Adjusted Hazard Ratios for Selected Clinical Outcomes in the Study Population during the 15-Year Study Period, According to the Fasting Glucose Category at Baseline and the Model.*

\begin{tabular}{|c|c|c|c|}
\hline Outcome & Model lb & Model 2b & Model 3b \\
\hline \multicolumn{4}{|l|}{ Diagnosed diabetes $\hat{\dagger}$} \\
\hline \multicolumn{4}{|l|}{ Fasting glucose category — hazard ratio $(95 \% \mathrm{Cl})$} \\
\hline$<100$ mg/dl (reference) & 1.00 & 1.00 & 1.00 \\
\hline 100 to $<126 \mathrm{mg} / \mathrm{dl}$ & $3.01(2.69-3.37)$ & $2.31(2.06-2.59)$ & $2.19(1.95-2.45)$ \\
\hline$\geq 126 \mathrm{mg} / \mathrm{dl}$ & $21.5(18.7-24.6)$ & $12.3(10.7-14.2)$ & 8.07 (6.92-9.42) \\
\hline$P$ value for trend & $<0.001$ & $<0.001$ & $<0.001$ \\
\hline $\begin{array}{l}\text { Fasting glucose - hazard ratio }(95 \% \mathrm{Cl}) \text { per } \\
\qquad 10 \mathrm{mg} / \mathrm{dl} \text { increase }\end{array}$ & $1.244(1.233-1.254)$ & $1.202(1.191-1.214)$ & $1.088(1.063-1.112)$ \\
\hline C statistic & 0.7546 & 0.7749 & 0.7816 \\
\hline
\end{tabular}

\section{Coronary heart disease}

Fasting glucose category - hazard ratio $(95 \% \mathrm{Cl})$

$$
\begin{aligned}
& <100 \mathrm{mg} / \mathrm{dl} \text { (reference) } \\
& 100 \text { to }<126 \mathrm{mg} / \mathrm{dl} \\
& \geq 126 \mathrm{mg} / \mathrm{dl}
\end{aligned}
$$

$P$ value for trend

Fasting glucose - hazard ratio $(95 \% \mathrm{Cl})$ per

$$
10 \mathrm{mg} / \mathrm{dl} \text { increase }
$$

C statistic

$\begin{array}{ccc}1.00 & 1.00 & 1.00 \\ 1.19(1.05-1.35) & 1.03(0.91-1.18) & 1.01(0.88-1.14) \\ 1.80(1.46-2.22) & 1.29(1.04-1.61) & 1.00(0.77-1.30) \\ <0.001 & 0.09 & 0.97 \\ 1.058(1.034-1.082) & 1.013(0.986-1.041) & 0.913(0.877-0.950) \\ 0.6761 & 0.7329 & 0.7383\end{array}$

\section{Ischemic stroke}

Fasting glucose category - hazard ratio $(95 \% \mathrm{Cl})$

$$
\begin{aligned}
& <100 \mathrm{mg} / \mathrm{dl} \text { (reference) } \\
& 100 \text { to }<126 \mathrm{mg} / \mathrm{dl} \\
& \geq 126 \mathrm{mg} / \mathrm{dl}
\end{aligned}
$$

$P$ value for trend

Fasting glucose - hazard ratio $(95 \% \mathrm{Cl})$ per

$$
10 \mathrm{mg} / \mathrm{dl} \text { increase }
$$

C statistic

2.

$\begin{array}{lll}1.00 & 1.00 & 1.00\end{array}$

$1.06(0.84-1.34) \quad 0.97(0.76-1.23) \quad 0.93(0.73-1.18)$

$2.33(1.68-3.24) \quad 1.89(1.33-2.69) \quad 1.30(0.85-1.98)$

$\begin{array}{lll}<0.001 & 0.02 & 0.63\end{array}$

$1.089(1.057-1.121) \quad 1.068(1.034-1.104) \quad 0.950(0.893-1.012)$

Death from any cause

Fasting glucose category — hazard ratio $(95 \% \mathrm{Cl})$

$$
<100 \mathrm{mg} / \mathrm{dl} \text { (reference) }
$$

0.7506

0.7594

$$
\begin{aligned}
& 100 \text { to }<126 \mathrm{mg} / \mathrm{dl} \\
& \geq 126 \mathrm{mg} / \mathrm{dl}
\end{aligned}
$$

$P$ value for trend

Fasting glucose - hazard ratio $(95 \% \mathrm{Cl})$ per $10 \mathrm{mg} / \mathrm{dl}$ increase

C statistic

$$
0.7109
$$

$\begin{array}{ccc}1.00 & 1.00 & 1.00 \\ 1.11(0.99-1.24) & 1.07(0.96-1.21) & 1.06(0.94-1.19) \\ 1.42(1.17-1.73) & 1.31(1.07-1.61) & 1.16(0.91-1.47) \\ 0.001 & 0.03 & 0.20 \\ 1.035(1.012-1.058) & 1.021(0.997-1.045) & 0.980(0.945-1.018) \\ 0.6865 & 0.7313 & 0.7314\end{array}$

* Model $\mathrm{lb}$ was adjusted for age, sex, and race (black or white). Model $2 \mathrm{~b}$ was adjusted for the variables in model $1 \mathrm{~b}$ plus low-density and high-density lipoprotein cholesterol levels, log-transformed triglyceride level, body-mass index, waist-to-hip ratio, hypertension (yes or no), family history of diabetes (yes or no), education (less than high school, high school or equivalent, or college or above), alcohol use (currently, formerly, or never), physical-activity index score, and smoking status (current smoker, former smoker, or never smoked). Model $3 b$ was adjusted for all variables in model $2 \mathrm{~b}$ plus the glycated hemoglobin value. To convert the values for fasting glucose to millimoles per liter, multiply by 0.05551 .

$\uparrow$ Diagnosed diabetes was defined on the basis of a self-reported diabetes diagnosis or diabetes medication use during 15 years of follow-up. 
all available for the first 6 years of the follow-up period - our results were similar (see the Supplementary Appendix). Owing to the observational nature of our investigation, the possibility of residual confounding cannot be completely eliminated. Nonetheless, this effort is one of the largest, community-based studies of glycated hemoglobin involving information about the development of diabetes and validated cardiovascular events during follow-up. Major strengths of this ARIC ancillary study were the use of comprehensive surveillance and adjudication of cardiovascular events and rigorous measurement of risk factors. The large population of blacks was an additional strength, permitting us to conduct robust analyses of possible racial differences in risk.
In this community-based study population of black or white nondiabetic adults, glycated hemoglobin was superior to fasting glucose for assessment of the long-term risk of subsequent cardiovascular disease, especially at values above $6.0 \%$. Such prognostic data may add to the evidence supporting the use of glycated hemoglobin as a diagnostic test for diabetes.

Supported by grants from the National Institutes of Health, National Institute of Diabetes and Digestive and Kidney Diseases (NIDDK) (R21 DK080294 and K01 DK076595, to Dr. Selvin; and K24 DK62222, to Dr. Brancati); contracts with the National Heart, Lung, and Blood Institute (N01-HC-55015, N01-HC-55016, N01-HC-55018, N01-HC-55019, N01-HC-55020, N01-HC-55021, and N01-HC-55022); and an NIDDK grant (P60 DK079637, to Dr. Brancati through the Johns Hopkins Diabetes Research and Training Center).

Financial and other disclosures provided by the authors are available with the full text of this article at NEJM.org.

\section{REFERENCES}

1. American Diabetes Association. Standards of medical care in diabetes - 2009. Diabetes Care 2009;32:Suppl 1:S13-S61.

2. Idem. Diagnosis and classification of diabetes mellitus. Diabetes Care 2009;32: Suppl 1:S62-S67.

3. American Diabetes Association. Diagnosis and classification of diabetes mellitus. Diabetes Care 2010;33:Suppl 1:S62-S69.

4. Selvin E, Crainiceanu CM, Brancati FL, Coresh J. Short-term variability in measures of glycemia and implications for the classification of diabetes. Arch Intern Med 2007;167:1545-51.

5. Phillipou G, Phillips PJ. Intraindividual variation of glycohemoglobin: implications for interpretation and analytical goals. Clin Chem 1993;39:2305-8.

6. Rohlfing C, Wiedmeyer HM, Little R, et al. Biological variation of glycohemoglobin. Clin Chem 2002;48:1116-8.

7. Selvin E, Zhu H, Brancati FL. Elevated A1C in adults without a history of diabetes in the U.S. Diabetes Care 2009;32:828-33. 8. Herman WH, Dungan KM, Wolffenbuttel $\mathrm{BH}$, et al. Racial and ethnic differences in mean plasma glucose, hemoglobin A1c, and 1,5-anhydroglucitol in over 2000 patients with type 2 diabetes. J Clin Endocrinol Metab 2009;94:1689-94.

9. Herman $\mathrm{WH}, \mathrm{Ma} \mathrm{Y}$, Uwaifo G, et al. Differences in A1C by race and ethnicity among patients with impaired glucose tolerance in the Diabetes Prevention Program. Diabetes Care 2007;30:2453-7.

10. Kirk JK, D'Agostino RB Jr, Bell RA, et al. Disparities in HbA1c levels between African-American and non-Hispanic white adults with diabetes: a meta-analysis. Diabetes Care 2006;29:2130-6.

11. The Atherosclerosis Risk in Communities (ARIC) Study: design and objectives. Am J Epidemiol 1989;129:687-702.

12. Jackson R, Chambless LE, Yang K, et al. Differences between respondents and nonrespondents in a multicenter community-based study vary by gender ethnicity. J Clin Epidemiol 1996;49:1441-6.

13. Duncan BB, Schmidt MI, Pankow JS, et al. Low-grade systemic inflammation and the development of type 2 diabetes: the Atherosclerosis Risk in Communities Study. Diabetes 2003;52:1799-805.

14. Siedel J, Hägele EO, Ziegenhorn J, Wahlefeld AW. Reagent for the enzymatic determination of serum total cholesterol with improved lipolytic efficiency. Clin Chem 1983;29:1075-80.

15. Nagele U, Hägele EO, Sauer G, et al. Reagent for the enzymatic determination of serum total triglycerides with improved lipolytic efficiency. J Clin Chem Clin Biochem 1984;22:165-74.

16. Friedewald WT, Levy RI, Fredrickson DS. Estimation of the concentration of low-density lipoprotein cholesterol in plasma, without use of the preparative ultracentrifuge. Clin Chem 1972;18:499-502.

17. Atherosclerosis Risk in Communities Coordinating Center. Operations manual no. 10: clinical chemistry determinations, version 1.0. Chapel Hill: University of North Carolina School of Public Health, 1987.

18. Idem. Operations manual no. 2: cohort component procedures, version 1.0. Chapel Hill: University of North Carolina School of Public Health, 1987.

19. Idem. Operations manual no. 11: sitting blood pressure, version 1.0. Chapel Hill: University of North Carolina School of Public Health, 1987.

20. Baecke JA, Burema J, Frijters JE. A short questionnaire for the measurement of habitual physical activity in epidemiological studies. Am J Clin Nutr 1982;36:936-42.

21. Rosamond WD, Folsom AR, Chambless LE, et al. Stroke incidence and sur- vival among middle-aged adults: 9-year follow-up of the Atherosclerosis Risk in Communities (ARIC) cohort. Stroke 1999; 30:736-43.

22. White AD, Folsom AR, Chambless LE, et al. Community surveillance of coronary heart disease in the Atherosclerosis Risk in Communities (ARIC) Study: methods and initial two years' experience. J Clin Epidemiol 1996;49:223-33.

23. Harrell FE Jr, Lee KL, Mark DB. Multivariable prognostic models: issues in developing models, evaluating assumptions and adequacy, and measuring and reducing errors. Stat Med 1996;15:361-87.

24. Pencina MJ, D'Agostino RB, D'Agostino RB Jr, Vasan RS. Evaluating the added predictive ability of a new marker: from area under the ROC curve to reclassification and beyond. Stat Med 2008;27:157-72. 25. Meigs JB, Nathan DM, Cupples LA, Wilson PW, Singer DE. Tracking of glycated hemoglobin in the original cohort of the Framingham Heart Study. J Clin Epidemiol 1996;49:411-7.

26. Engelgau MM, Geiss LS, Saaddine JB, et al. The evolving diabetes burden in the United States. Ann Intern Med 2004;140: 945-50.

27. Bertoni AG, Krop JS, Anderson GF, Brancati FL. Diabetes-related morbidity and mortality in a national sample of U.S. elders. Diabetes Care 2002;25:471-5.

28. Stout RL, Fulks M, Dolan VF, Magee ME, Suarez L. Relationship of hemoglobin A1c to mortality in nonsmoking insurance applicants. J Insur Med 2007;39:17481.

29. Pradhan AD, Rifai N, Buring JE, Ridker PM. Hemoglobin A1c predicts diabetes but not cardiovascular disease in nondiabetic women. Am J Med 2007;120:720-7. 30. de Vegt F, Dekker JM, Ruhé HG, et al. Hyperglycaemia is associated with all-cause 
and cardiovascular mortality in the Hoorn population: the Hoorn Study. Diabetologia 1999;42:926-31.

31. Blake GJ, Pradhan AD, Manson JE, et al. Hemoglobin A1c level and future cardiovascular events among women. Arch Intern Med 2004;164:757-61.

32. Khaw KT, Wareham N, Bingham S, Luben R, Welch A, Day N. Association of hemoglobin A1c with cardiovascular disease and mortality in adults: the European prospective investigation into cancer in Norfolk. Ann Intern Med 2004;141:413-20. 33. Brewer N, Wright CS, Travier N, et al. A New Zealand linkage study examining the associations between A1C concentration and mortality. Diabetes Care 2008;31: 1144-9.

34. Levitan EB, Liu S, Stampfer MJ, et al. HbA1c measured in stored erythrocytes and mortality rate among middle-aged and older women. Diabetologia 2008;51:267-75. 35. Meigs JB, Nathan DM, D'Agostino RB, Wilson PW. Fasting and postchallenge glycemia and cardiovascular disease risk: the Framingham Offspring Study. Diabe tes Care 2002;25:1845-50.
36. Gerstein HC, Swedberg K, Carlsson J, et al. The hemoglobin A1c level as a progressive risk factor for cardiovascular death, hospitalization for heart failure, or death in patients with chronic heart failure: an analysis of the Candesartan in Heart Failure: Assessment of Reduction in Mortality and Morbidity (CHARM) program. Arch Intern Med 2008;168:1699-704

37. Selvin E, Coresh J, Shahar E, Zhang L, Steffes M, Sharrett AR. Glycaemia (haemoglobin A1c) and incident ischaemic stroke: the Atherosclerosis Risk in Communities (ARIC) Study. Lancet Neurol 2005;4: 821-6.

38. Selvin E, Coresh J, Golden SH, Brancati FL, Folsom AR, Steffes MW. Glycemic control and coronary heart disease risk in persons with and without diabetes: the Atherosclerosis Risk in Communities Study. Arch Intern Med 2005;165:1910-6.

39. The Action to Control Cardiovascular Risk in Diabetes Study Group. Effects of intensive glucose lowering in type 2 diabetes. N Engl J Med 2008;358:2545-59.

40. Home PD, Pocock SJ, Beck-Nielsen H, et al. Rosiglitazone evaluated for cardio- vascular outcomes - an interim analysis. N Engl J Med 2007;357:28-38.

41. The ADVANCE Collaborative Group. Intensive blood glucose control and vascular outcomes in patients with type 2 diabetes. N Engl J Med 2008;358:2560-72.

42. Nissen SE, Wolski K. Effect of rosiglitazone on the risk of myocardial infarction and death from cardiovascular causes. N Engl J Med 2007;356:2457-71. [Erratum, N Engl J Med 2007;357:100.]

43. Selvin E, Bolen S, Yeh HC, et al. Cardiovascular outcomes in trials of oral diabetes medications: a systematic review. Arch Intern Med 2008;168:2070-80.

44. The Diabetes Control and Complications Trial Research Group. Design and methodologic considerations for the feasibility phase. Diabetes 1986;35:530-45.

45. The UK Prospective Diabetes Study (UKPDS) Group. Intensive blood-glucose control with sulphonylureas or insulin compared with conventional treatment and risk of complications in patients with type 2 diabetes (UKPDS 33). Lancet 1998;352:837-53. [Erratum, Lancet 1999;354:602.]

Copyright (c) 2010 Massachusetts Medical Society.

POSTING PRESENTATIONS AT MEDICAL MEETINGS ON THE INTERNET

Posting an audio recording of an oral presentation at a medical meeting on the Internet, with selected slides from the presentation, will not be considered prior publication. This will allow students and physicians who are unable to attend the meeting to hear the presentation and view the slides. If there are any questions about this policy, authors should feel free to call the Journal's Editorial Offices. 